\title{
Calcifying Pseudoneoplasm of the Neuraxis with Single Nerve Rootlet Involvement
}

\author{
Mark E. Jentoft, Bernd W. Scheithauer, Franco Bertoni, Christopher Abood, Howard T. Chang
}

Can J Neurol Sci. 2012; 39: 840-842

Calcifying pseudoneoplasm of the neuraxis (CPN) is also known by several other names including the catch-all "fibroosseous lesion," as well as "calcifying pseudotumor of the neural axis". Grossly, it is usually well-circumscribed, gritty or hard in consistency and up to several centimeters in size. Histologically, it consists largely of relatively amorphous material surrounded in part by a layer of mesenchymal-appearing cells variably immunoreactive for vimentin and EMA (epithelial membrane antigen). Calcification within the amorphous material is a frequent finding giving the material a chondrocalcific appearance. Metaplastic bone formation often occurs, even in lesions remote from bone. On occasion, multinucleated giant cells and even adipose tissue may be seen. Overall, CPN is considered to be a benign, reactive process with morbidities related to its local effects.

\section{Case Report}

The patient, a 26-year-old female, presented with left-sided and lower back pain. She had no known previous injury to her back. A magnetic resonance imaging (MRI) scan was performed which demonstrated a small $0.5 \times 0.4 \times 0.3-\mathrm{cm}, \mathrm{T}-2$ hypointense, intradural nodule in the left lateral thecal sac at level L1-2 that involved the left nerve roots. The radiologic impression was that of a small schwannoma. A repeat MRI scan 3.5 months later demonstrated relative stability of the now $0.8 \times 0.5 \times 0.5-\mathrm{cm}$ lesion (Figure 1). One month thereafter, the patient underwent a gross total resection. At surgery, the mass was noted to be firm, red-tan and involved a single spinal rootlet. The lesion was removed en bloc. Grossly, it measured $0.8 \times 0.5 \times 0.5 \mathrm{~cm}$, was well circumscribed, white and gritty on cut surface and featured a small nerve entering one end and exiting the other. The frozen section interpretation favored a low-grade tumor with dystrophic calcification.
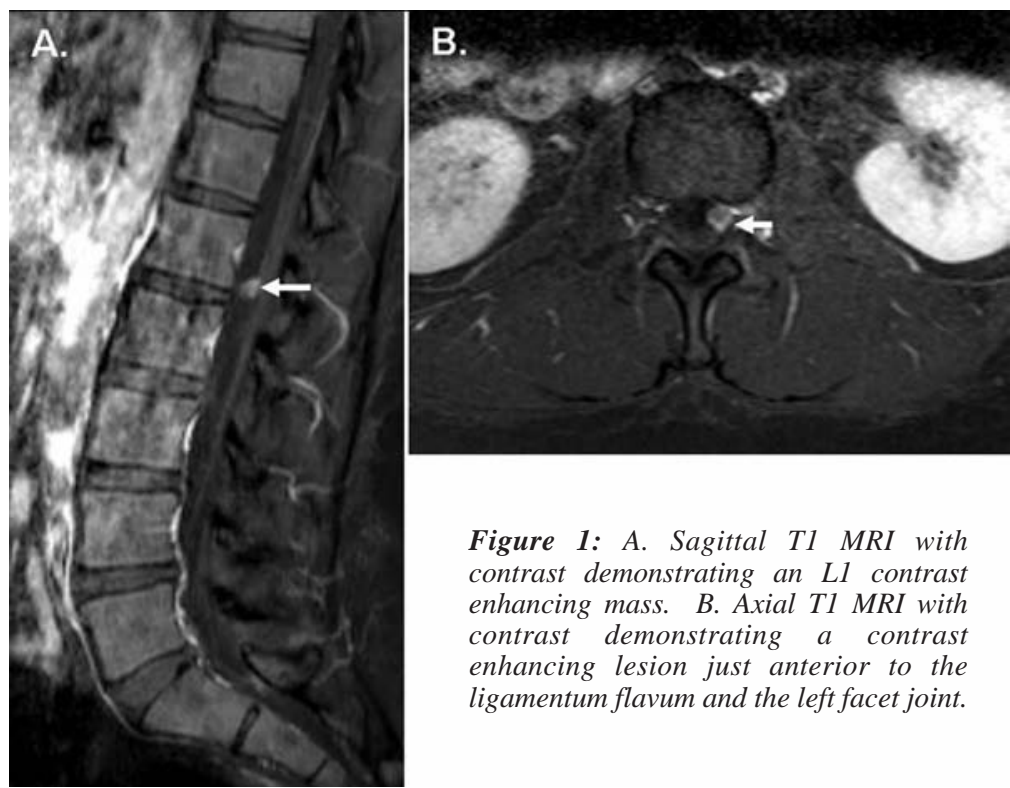

Figure 1: A. Sagittal T1 MRI with contrast demonstrating an L1 contrast enhancing mass. B. Axial T1 MRI with contrast demonstrating a contrast enhancing lesion just anterior to the ligamentum flavum and the left facet joint.

\footnotetext{
From the Departments of Neuropathology (MEJ), Laboratory Medicine and Anatomic Pathology (BWS), Mayo Clinic, Rochester, MN; Lansing Neurosurgery (CA); Lansing; Neurology \& Ophthalmology (HTC), Michigan State University, East Lansing, Michigan, USA; Department of Pathology (FB), Villa Erbosa Hospital, Bologna, Italy; Received August 30, 2011. Final Revisions Submitted June 14, 2012. 


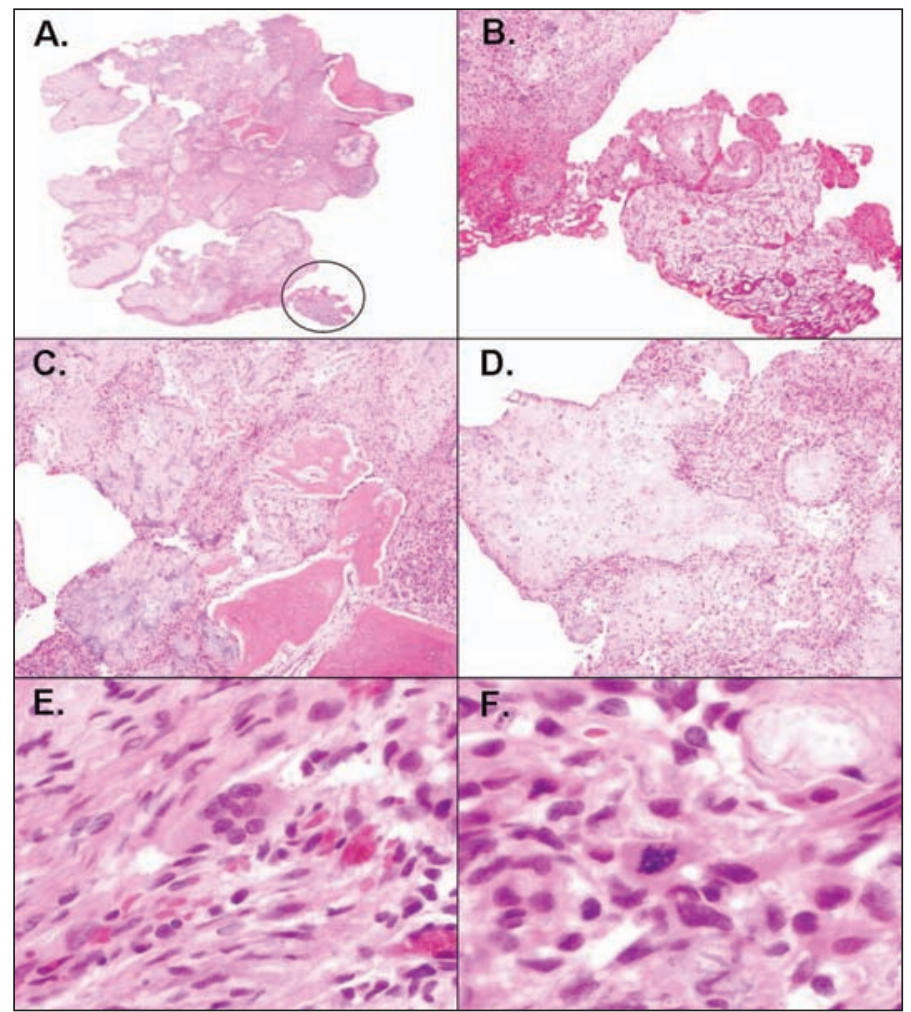

Figure 2: $A$. $H \& E$ 1.25X. View of entire lesion demonstrating the heterogeneous nature of the mass, the nerve rootlet is circled. B. H\&E 10X. View of the nerve rootlet. C. $H \& E \quad 10 X$. Demonstrating the heterogeneity of the lesion with basophilic amorphous areas, regions with increased cellularity, and bone. D. H\&E 10X. Clear delineation is demonstrated between the relatively amorphous regions and the areas of increased cellularity, a rind of cells is noted surrounding the amorphous areas. $E$ and $F$. $H \& E$ 40X. A multinucleated giant cell and mitotic figure, respectively.

On microscopic review, the lesion was noted to be intimately associated with a segment of spinal nerve root. Its heterogenous histologic appearance comprised three distinct patterns including: a) hypocellular amorphous matrix, b) encircling cellular zones, and c) metaplastic bone. The hypocellular element, basophilic and chondrocalcific in appearance, had a delicate, somewhat fibrillary or brushed appearance. This was particularly the case in its peripheral zones where cytologically benign, epithelioid to spindle-shaped cells were arrayed around the hypocellular areas to form a rind. The bone present appeared entirely benign and metaplastic in nature, being generally separated from the hypocellular amorphous matrix. The intervening cellular areas contained foci of meningothelial cells, blood vessels, plump spindled cells and rare giant cells. A single mitotic figure was noted within one of these intervening cellular regions (Figure 2).

Immunohistochemical stains were performed for EMA (Dako; Carpinteria, CA; clone E29, 1:50 BRD), S-100 (Dako, polyclonal, 1:1600), neurofilament (NF) (Dako, clone 2F11, 1:800 BRD), and smooth muscle actin (SMA) (Dako, clone 14A, $1: 250)$. The rind of cells that surround the hypocellular regions stained positively for EMA which appears distinct in this case from the stromal cells which lack significant EMA reactivity (Figure 3). S-100 and NF highlighted not only the portion of

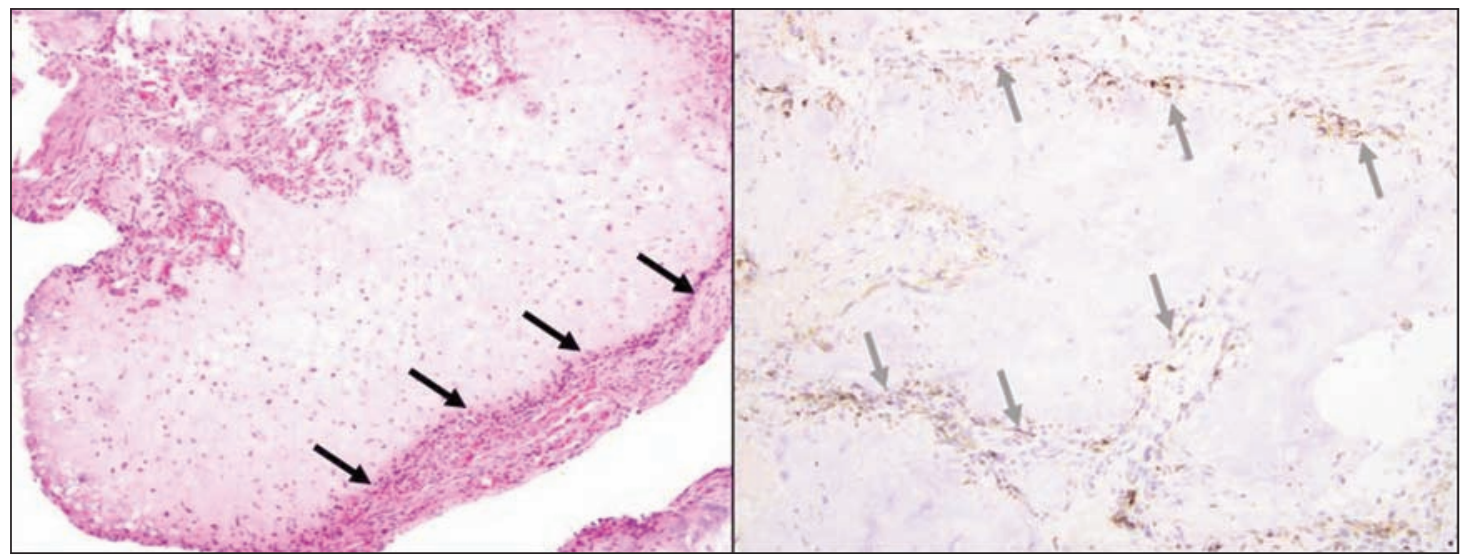

Figure 3: $H \& E$ and EMA 10x. The arrows highlight a rind of cells surrounding the amorphous areas, these cells stain positively for EMA. 
intact nerve present but also small axonal bundles present multifocally within the substance of the lesion (Figure 4). SMA stained positively within the cellular areas of the lesion but was not positive in the cells delimiting the hypocellular concretions. Based on the overall features of the lesion, a diagnosis of calcifying pseudoneoplasm of the neuraxis was made. The patient's postoperative course was uneventful and she had alleviation of her symptoms.

\section{Discussion}

Calcifying pseudoneoplasm of the neuraxis was first reported in 1978 as an "unusual fibro-osseous lesion" in the central nervous system ${ }^{1}$. Its rarity is highlighted in that the largest reported case series consists of only 14 cases and spans a 36-year period in the high-volume institutional and consultative service of the Mayo Clinic (1950-1986) ${ }^{2}$. The last comprehensive review of the subject in 1999 discusses 27 cases, inclusive of all reported to date ${ }^{3}$. Average patient age at diagnosis was 46 years (range, 12-83) with a slight male predominance $\left(1.6{ }^{3}: 1.0\right.$ 우 $)$ Sixteen tumors $(60 \%)$ were intracranial (10 extra-axial and 6 intra-axial); 11 were intraspinal-extra-axial, dural-based and distributed in the upper cervical to lower lumbar regions ${ }^{3}$. Since then, additional cases have been reported.

These lesions are felt to be benign, reactive processes in which complete excision is curative. Despite their indolent nature, they are sometimes locally destructive and can be clinically problematic due to location, specifically at the skull base whereat mortality has been reported ${ }^{2}$. It is advisable that clinical follow-up is provided in cases in which complete surgical removal of the lesion cannot be achieved, especially if the patients symptoms worsen or the lesion involves or is adjacent to critical structures. Calcifying pseudoneoplasm has only occasionally been reported to arise in intimate association with other pathologic processes, specifically meningioangiomatosis, a lesion associated with neurofibromatosis, type 2 $(\mathrm{NF} 2)^{4}$. Additionally, one of the authors (BWS) has observed and previously reported a case of $\mathrm{CPN}$ associated with an ependymoma and a case of CPN overlying a dysembryoplastic neuroepithelial tumor. Though calcium deposition occurs within CPNs, no clear association with a hypercalcemic state has been established.

Calcifying pseudoneoplasm of the neuraxis has been theorized to arise either from arachnoid cells due to their frequent association with leptomeninges and dura, or from fibroblasts or other mesenchymal cells due to their ultrastructure and immunophenotype ${ }^{3}$. A histologic similarity to tumefactive processes found at other locations such as calcifying aponeurotic fibroma, supports the latter interpretation ${ }^{3}$. Our case supports the arachnoidal nature of at least some, if not all, neuraxis examples. Our case featured discrete involvement by CPN of a single spinal nerve rootlet within the lumbar region, the resultant symptoms being pain and lower extremity paresthesia. Axon bundles and accompanying Schwann cells were multifocally identified within the substance of the lesion which highlights this case's relationship to the nerve root. Though clinically this case has features similar to neoplasms such as a schwannoma or meningioma, the histopathologic characteristics of this lesion preclude these diagnoses. To our knowledge, a CPN of the neuraxis has not been previously described as involving a single

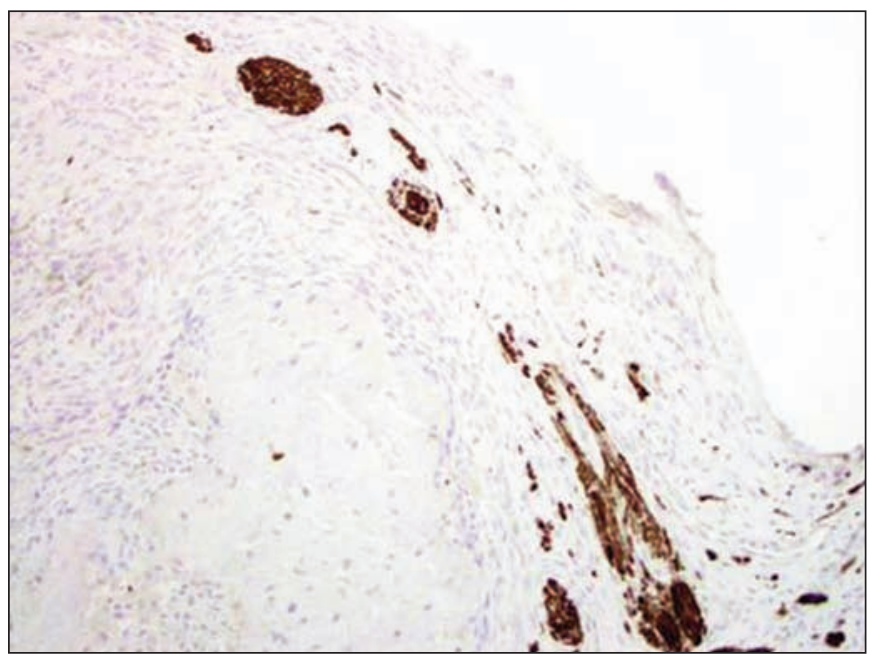

Figure 4: S100, 20X. An axon bundle within the substance of the pseudoneoplasm is highlighted.

nerve root in such an intimate, discriminating fashion with radiographic and gross features suggestive of a neoplasm. This being said, a similar reactive lesion with calcification and fibroblastic proliferation has been recently described in an intradural extramedullary location ${ }^{5}$. However, this did not have the described chondrocalcific appearance of a CPN nor bone formation. Despite the lack of bone formation these authors thought it was a reactive condition and compared it to neuritis ossificans, which is a disease of peripheral nerve caused by ossification of the nerve sheath ${ }^{6}$. If additional cases of CPN involving nerve roots are brought to light, perhaps a separate name should be entertained for this CPN subtype, such as "radiculitis calcificans", which describes both the clinical condition of radicular pain and its pathology. Though rare, CPN may enter into the differential diagnosis of spinal nerve root lesions, including tumors.

\section{REFERENCES}

1. Rhodes RH, Davis RL. An unusual fibro-osseous component in intracranial lesions. Hum Pathol. 1978;9(3):309-19.

2. Bertoni F, Unni KK, Dahlin DC, Beabout JW, Onofrio BM. Calcifying pseudoneoplasms of the neural axis. J Neurosurg. 1990;72(1):42-8.

3. Qian J, Rubio A, Powers JM, et al. Fibro-osseous lesions of the central nervous system: report of four cases and literature review. Am J Surg Pathol. 1999;23(10):1270-5.

4. Burger PC, Scheithauer BW. Tumors of the central nervous system (AFIP Atlas of Tumor Pathology. Fourth series; fasc. 7) Washington, D.C.: American Registry of Pathology in collaboration with the Armed Forces Institute of Pathology; 2007.

5. Apostolopoulos V, David KM, Malcolm A, King A. Intradural calcifying fibroblastic proliferation associated with a nerve root: a reactive process mimicking a nerve sheath tumor. Spine (Phila Pa 1976). 2009;34(19):E712-5.

6. George DH, Scheithauer BW, Spinner RJ, et al. Heterotopic ossification of peripheral nerve ("neuritis ossificans"): report of two cases. Neurosurgery. 2002;51(1):244-6; discussion 6 . 